
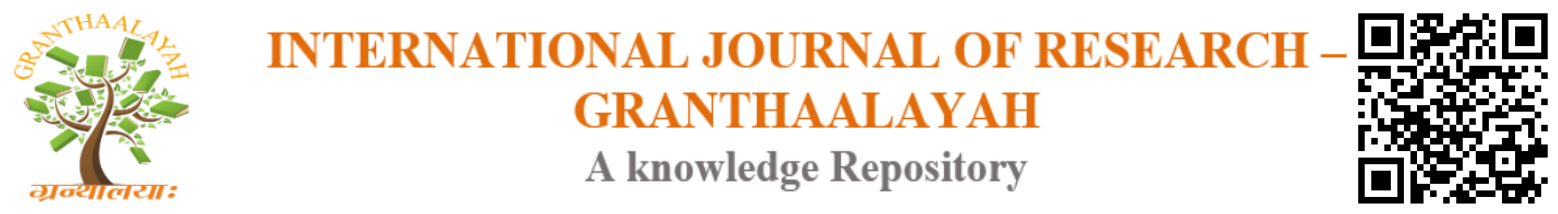

Science

\title{
BIOSYNTHESIS OF POLYHYDOXYALKANOATE
}

\author{
Ahmad Idi *1 \\ ${ }^{* 1}$ Department of Biological Sciences, Adamawa State University, Mubi, Nigeria
}

\begin{abstract}
Polydroxyalkanoate (PHA) is produced and stored as water-insoluble granules in the cytoplasm of many bacteria. It possesses similar physical properties to petroleum-based polyesters. Hence it is considered as degradable plastic that can replaced the petroleum-based polyesters. This article reviews the various processes involved in the biosynthesis, identification and characterisation of PHA. It covers both the presumptive and confirmatory test in the identification of PHA produced by bacteria as well as the various methods of PHA recovery. Techniques such as Nuclear Magnetic Resonance (NMR), Fourier Transform Infrared Spectroscopy (FTIR) and chromatography were discussed in relation to PHA characterisation. The identification of PHA producing bacteria and characterisation of PHA are significant for both quantitative and qualitative analysis of PHA.
\end{abstract}

Keywords: Polyhydroxylkanote; Chromatography; Microscopy; FTIR; NMR.

Cite This Article: Ahmad Idi. (2019). "BIOSYNTHESIS OF POLYHYDOXYALKANOATE." International Journal of Research - Granthaalayah, 7(7), 200-206. https://doi.org/10.29121/granthaalayah.v7.i7.2019.748.

\section{Introduction}

With the increasing challenges facing the use of fossil fuel and the rapid accumulation of plastic wastes in the environment, an alternative source that can substitute petroleum-based synthetic plastic becomes a focus of research nowadays. Plastics have universal application as construction and packaging materials, parts of automobile and home appliances, disposable utensil as well as in medicine [1]. Due to this versatility in usage and high demand, its annual growth rate has been reported at 9\% with an increment from 1.5 million tons in 1950 to 245 million in 2008 [2]. This leads to annual plastic waste accumulation of 25 million tons which require about 400 years to degrade [3].

Techniques such as landfill, recycling and incineration are considered ineffective in managing this waste. Apart from the release of greenhouse gases and other toxic compounds, incineration of plastics produces additives that may be carcinogenic [3]. Landfill results in polluting the surrounding environment while recycling plastics limits its applicability by changing its properties in addition to difficulty in sorting out different kinds of plastics [4]. The difficulty in managing this waste is attributed to the presence of large proportion of polyolefin that is recalcitrant to 
biodegradation due to its hydrophobicity, absence of active functional group, high molecular weight, presence of additives and its branched structure [5]. These features make microbial degradation of this pollutant unsuccessful.

Polyhydroxyalkanoates (PHA) are natural polyesters accumulated by some bacteria under growth limited condition as energy and carbon reserves. As such they are easily degraded in soil by bacteria that possess the enzyme (PHA polymerase), which catalyzes the ester bonds to its oligomers and water-soluble monomers. Microorganisms further metabolize these products into $\mathrm{H}_{2} \mathrm{O}$ and $\mathrm{CO}_{2}$ [2]. They are therefore renewable and biodegradable having similar characteristics with synthetic plastic. Their thermoplastic and biodegradable properties make them ideal candidate as an alternative to petroleum-based synthetic plastic. Other properties such as biocompatibility and elastomeric broadened their application to industries and medicine. In medicine they are used to develop cardiovascular patches, articular cartilage repair device and bone-marrow scaffolds [6] This diversity in application and functions of PHA are attributed to its possession of large number of monomers. The type and composition of these monomers are determined by the bacterial strain, growth condition and the relative amount of carbon source use in the media [7]. It is reported that PHA has over 150 different monomers produced by many different bacterial species [8] including Ralstonia eutropha, Pseudomonads, Azobacter chroococcum, methylotrophs, Alcaligenes latus[9]. Despite the large numbers of different monomers produced by many bacterial genera, only few types of PHA are being commercialized. The major challenges facing the commercialization of PHA among others include high production cost, low yield, limited number of PHA producing bacteria, existence of impurities and difficulty in recovery [10]. This article therefore reviews the various processes involved in the identification and characterisation of PHA.

\section{Structure of PHA}

Structurally PHA is a linear polyester joined by hydroxacyl units [11]. The functional alkyl R group described in Figure 1 determined the name and carbon number of the PHA while the asterisk described the chiral carbon which is the building block of the polymer. They are structurally classified into three groups based on the number of carbon atoms within the monomer. Short chain length possess 3-5 carbon atoms, medium chain possess 6-14 carbon atoms and long chain possess more than 15 carbon atoms[12]. The short chains have crystallinity of $60-80 \%$. They are also characterised by brittleness and stiffness as well as thermal instability. The medium chains have low crystallinity of $25 \%$, low tensile strength and melting temperature in addition of higher elongation to break [13]; [14].

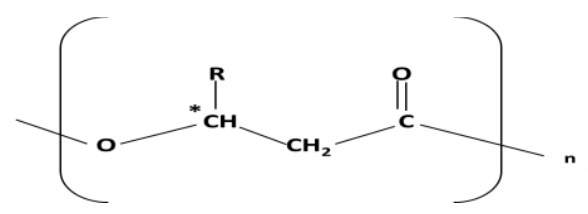

Figure 1: Chemical structure of PHA redrawn from [12]

\section{Biosynthetic Pathways of PHA}

The type and monomeric composition of PHA are determined by carbon source used for PHA synthesis. Hence three pathways are common reported for PHA biosynthesis. These pathways are described in Figure 2. In the first pathways, 2-acetyl-coA which is produced from either fatty acids, 
sugar or amino acid is converted to hydroxybutyryl-coA by the enzymes acetoacetyl-coA reductase and $\beta$-ketothiolase. This is later polymerized by PHA synthase to produced PHB. This pathway describes the production of scl PHA $\left(\mathrm{C}_{3}-\mathrm{C}_{5}\right)$. In the second pathway, fatty acids are converted to enoyl-coA which is subsequently converted to R-3-hydroxyacyl-coA by R3hydroxyacyl-coA hydratase. R-3-hydroxyacyl-coA is then catalyzed by PHA synthase to produced PHA. This pathway describes atypical mcl PHA synthesis. In the third pathways, the in situ fatty acid synthesis cycle is involved. Here, 3-hydroxyacyl-ACP is transform to 3hydroxyacyl-coA for the synthesis of PHA by the enzyme 3-hydroxyacyl-acyl carrier protein-coA tranferase.[15].

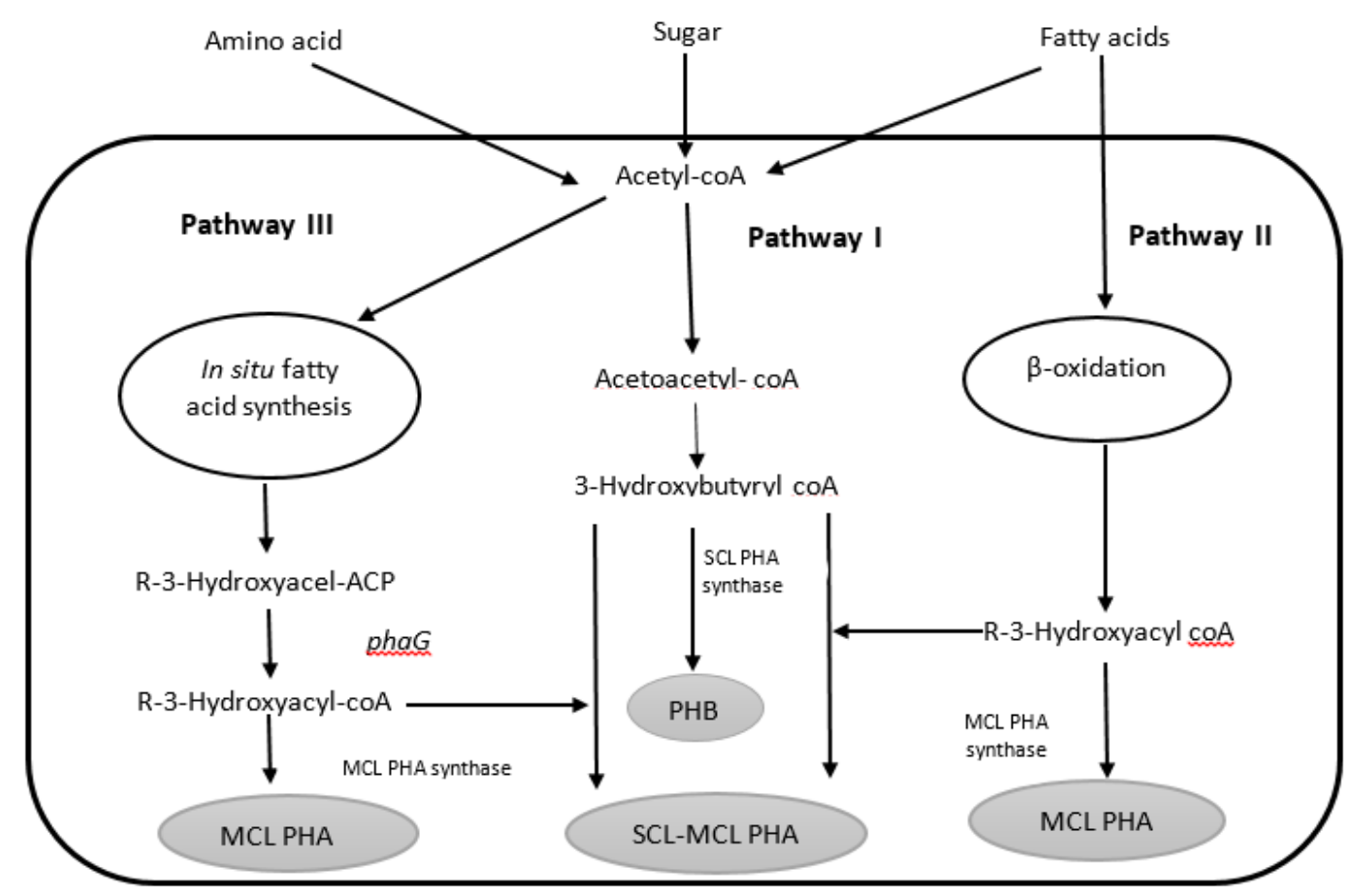

Figure 2: Biosynthetic pathways of PHA redrawn from[15].

\section{Recovery of PHA}

PHA as an intracellular material is always linked (attached) to the cell biomass. Their isolation (separation) and purification from the bacterial cell is a major challenge in industrial application [16]. Various strategies are therefore developed for the recovery of PHA. Steps such as lyophilisation, extraction, purification and hydrolysis are needed in the recovery of PHA for the purpose both quantitative and qualitative analysis as well as characterisation of PHA.

The use of solvent in recovery of PHA is the most widely method of PHA recovery due to its simplicity, high yield, high purity, limited PHA degradation and removal of endotoxin[17]. The application of organic solvent in PHA extraction is based on the concept that PHA is insoluble in water but soluble in some organic solvents [16]. During solvent extraction, the permeability of cell membrane is modified to release and solubilize the PHA and the precipitation of non-solvent is followed[17]. Among the various organic solvents, chloroform widely used in PHA extraction. 
The recovery with chloroform was reported by[18], in which about $3.0 \mathrm{~g}$ of lyophilized biomass was incubated at $30^{\circ} \mathrm{C}$ for $24 \mathrm{hr}$ before the chloroform was concentrated with rotary evaporator. A halogen-free method using $\mathrm{NaOH}$ and water with recovery yield of more than 90\% [19] was also established as an effective method of PHA extraction. Other methods such digestion method, supercritical fluid, mechanical disruption, cell fragility, chemical and enzymatic disruption also yield high amount of PHA [16].

\section{Methods of Detecting and Characterisation of PHA}

\subsection{Microscopy Examination of PHA}

PHA can be detected in intact cell biomass prior to extraction and visualized with light microscope using staining dye such as Sudan Black B and Oxazine dye (Nile Blue A and Nile Red) [13]. PHB inclusion was visualized by staining with Sudan Black B in mixed bacterial cell[20]. Screening of microalgae for PHB positive was also performed with Sudan Black B[21]. Hence the use of Sudan Black B staining technique for PHA detection was not only applicable to bacteria but also microalgae.

A simple and more sensitive staining technique for the detection of PHA using oxazine dye (Nile Blue A and Nile Red) was also reported. In contrast to the used of Sudan Black B, this method allows the dye to be incorporated directly into the fermentation medium at a concentration of $0.5 \mathrm{ug} / \mathrm{ml}$. It was observed that the presence of the dye had no effect on the growth and accumulation of PHA [22]. PHA-Producing bacteria appeared yellow/pink/red/orange under UV light when stain with Nile Blue A or Nile red. This appearance enables the PHA-producing bacteria to isolated and identified.

Transmission electron microscopy is also used to detect intracellular PHA granules. TEM details not only the cellular inclusion but also the structure of the bacteria. The micrographs of PHA granules when view with TEM have been shown to vary with different carbon sources and cultural condition as well as the bacterial strain [23].

\subsection{Crotonic Acid Assay}

The use of crotonic acid to quantify PHB is traditionally the common technique for quantification of PHB. It is based on the principle that PHB is converted to crotonic acid when dissolved in sulphuric acid and has a strong UV absorption at $234 \mathrm{~nm}$. Although this technique is simple and fast, it is only restricted to PHB and it is also reported to be ineffective in PHB quantification due to over estimation [24].

\subsection{Amplification of phaC Gene}

Another important method of detecting PHA is through the amplification of the phaC gene encoding the enzyme PHA synthase which is typically present in all PHA producing bacteria and absent in non PHA producing bacteria. This method has been reported to be more specific compared to the used of the laborious traditional staining techniques explained above. Additionally, the method does not depend on the usual unbalance growth condition of PHA 
production. As such it is applicable in normal growth condition because it specifically target the bacteria rather culture condition [25].

\subsection{Fourier Transform Infrared Spectroscopy}

The FTIR is used to detect PHA by the conformation of its functional groups in purified sample or in an intact cell. The method has also been reported as powerful tool for screening and quantification of PHA [26] as well determine the degree its crystallisation [27]. The advantage of this method is short time analysis with fast data output and does not require solvent, so eliminate the cost and risk of chemical exposure[12]. However, this method is more suitable in characterisation of PHA rather than detection because of its inability to detect low PHA content and differentiating between blend and copolymer[28].

\subsection{Nuclear Magnetic Resonance (NMR)}

NMR is also an important tool used to confirm the identity of PHA. It is significant in structural characterisation of PHA before being hydrolyse to determine whether it is a copolymer or homopolymer[29]. It has also been shown to be an effective tool for the quantification of PHA from full-strength wastewater containing organic compounds with high concentration of impurities [30]. The NMR spectrum assigned absorption area to a nuclei of a molecule in different electronic and chemical environments. The assigned absorption area is proportional to the corresponding number of nuclei. This give useful data about the molecule [31].

\subsection{Chromatographic-Based Techniques of PHA Characterisation}

Analytical techniques such as GC and HPLC are useful techniques that provide both qualitative and quantitative information about the monomeric composition of PHA. These techniques provide more sensitive detection analysis of PHA compared to other methods[12]. However, these techniques require additional step such as hydrolysis prior to analysis. It is also depended on the use of appropriate PHA analytical standards. Furthermore the conventional chromatographicbased technique of PHA quantification is shown to be inappropriate for medium-chain length PHA [32]. HPLC has also been reported to provide only quantitative information about PHA but its ability to provide qualitative information is limited [33]. However, an advanced technology such as GCMS and LCMS which do not require a standard and are more sensitive and accurate in detecting various monomers of PHA were developed recently. For instance, GCMS is reported to be more specific and accurate in providing both qualitative and quantitative information about the monomeric structure of PHA [33].

\section{Thermal Analysis of PHA}

The thermal characteristic of PHA reveals information about the changes that occur in PHA composition with temperature. This is measured by DSC or TGA. The difference between the two is that TGA measures changes in weight during heating while DSC measures heat liberated or absorb during heating. The standard method of running DSC is at a temperature from $-100^{\circ} \mathrm{C}$ to $400^{\circ} \mathrm{C}$ while TGA is run from room temperature to $700^{\circ} \mathrm{C}$. Both TGA and DSC are performed at the heating rate of $10^{\circ} \mathrm{C} \mathrm{min}^{-1}$ under controlled air or $\mathrm{N}_{2}$ [29]. 


\section{Conclusion}

Commercialisation of PHA required the identification of more PHA producing bacteria, removal of impurities and cheap solvents for PHA recovery among others. These factors have been shown to affect the physical properties of PHA. Hence various characterisation techniques are required to ascertain the properties of PHA. The quality and quantity of PHA are of great significant to polymer scientists.

\section{References}

[1] Castilho, L.R., D.A. Mitchell, and D.M. Freire, Production of polyhydroxyalkanoates (PHAs) from waste materials and by-products by submerged and solid-state fermentation. Bioresource technology, 2009. 100(23): p. 5996-6009.

[2] Chanprateep, S., Current trends in biodegradable polyhydroxyalkanoates. Journal of bioscience and bioengineering, 2010. 110(6): p. 621-632.

[3] Preeti, C. and T. Archana, Integration of natural and Biological sources for the Production of Biopolymer: Actual and Potential Utilization of various Wastes. Journal of Pharmacy Research, 2011. 4(1).

[4] Oehlmann, J., et al., A critical analysis of the biological impacts of plasticizers on wildlife. Philosophical Transactions of the Royal Society B: Biological Sciences, 2009. 364(1526): p. 2047 2062.

[5] Arutchelvi, J., et al., Biodegradation of polyethylene and polypropylene. Indian Journal of Biotechnology, 2008. 7(1): p. 9.

[6] Valappil, S.P., et al., Biomedical applications of polyhydroxyalkanoates, an overview of animal testing and in vivo responses. Expert Review of Medical Devices, 2006. 3(6): p. 853-868.

[7] Ojumu, T., J. Yu, and B. Solomon, Production of polyhydroxyalkanoates, a bacterial biodegradable polymers. African Journal of Biotechnology, 2004. 3(1): p. 18-24.

[8] Hazer, B. and A. Steinbüchel, Increased diversification of polyhydroxyalkanoates by modification reactions for industrial and medical applications. Applied Microbiology and Biotechnology, 2007. 74(1): p. 1-12.

[9] Panchal, B., A. Bagdadi, and I. Roy, Polyhydroxyalkanoates: the natural polymers produced by bacterial fermentation, in Advances in natural polymers. 2013, Springer. p. 397-421.

[10] Tokiwa, Y. and C.U. Ugwu, Biotechnological production of (R)-3-hydroxybutyric acid monomer. Journal of biotechnology, 2007. 132(3): p. 264-272.

[11] Peña, C., et al., Biotechnological strategies to improve production of microbial poly-(3hydroxybutyrate): a review of recent research work. Microbial biotechnology, 2014. 7(4): p. 278293.

[12] Tan, G.-Y.A., et al., Start a research on biopolymer polyhydroxyalkanoate (PHA): a review. Polymers, 2014. 6(3): p. 706-754.

[13] Anjum, A., et al., Microbial production of polyhydroxyalkanoates (PHAs) and its copolymers: A review of recent advancements. International journal of biological macromolecules, 2016. 89: p. 161-174.

[14] Zhao, Y.-X.X., et al., Poly(3-hydroxybutyrate-co-3-hydroxyvalerate) production by Haloarchaeon Halogranum amylolyticum. Applied microbiology and biotechnology, 2015. 99(18): p. 7639-7649.

[15] Chen, G.-Q., et al., Engineering biosynthesis mechanisms for diversifying polyhydroxyalkanoates. Trends in biotechnology, 2015. 33(10): p. 565-574.

[16] Madkour, M.H., et al., PHA recovery from biomass. Biomacromolecules, 2013. 14(9): p. 29632972.

[17] Kunasundari, B. and K. Sudesh, Isolation and recovery of microbial polyhydroxyalkanoates. Express Polym. Lett, 2011. 5(7): p. 620-634. 
[18] Zakaria, M.R., et al., Biosynthesis and characterization of poly (3-hydroxybutyrate-co-3hydroxyvalerate) copolymer from wild-type Comamonas sp. EB172. Polymer Degradation and Stability, 2010. 95(8): p. 1382-1386.

[19] Mohammadi, M., et al., Intracellular polyhydroxyalkanoates recovery by cleaner halogen-free methods towards zero emission in the palm oil mill. Journal of Cleaner Production, 2012. 37: p. 353-360.

[20] Cavaillé, L., et al., Understanding of polyhydroxybutyrate production under carbon and phosphorus-limited growth conditions in non-axenic continuous culture. Bioresource technology, 2016. 201: p. 65-73.

[21] Kavitha, G., et al., Biosynthesis, purification and characterization of polyhydroxybutyrate from Botryococcus braunii kütz. International Journal of Biological Macromolecules, 2016. 89: p. 700706.

[22] Spiekermann, P., et al., A sensitive, viable-colony staining method using Nile red for direct screening of bacteria that accumulate polyhydroxyalkanoic acids and other lipid storage compounds. Archives of Microbiology, 1999. 171(2): p. 73-80.

[23] Yoneyama, F., et al., Production of polyhydroxybutyrate and alginate from glycerol by Azotobacter vinelandii under nitrogen-free conditions. Bioengineered, 2015. 6(4): p. 209-217.

[24] Valappil, S.P., et al., Polyhydroxyalkanoates in Gram-positive bacteria: insights from the genera Bacillus and Streptomyces. Antonie Van Leeuwenhoek, 2007. 91(1): p. 1-17.

[25] Romo, D., et al., A most effective method for selecting a broad range of short and medium-chainlength polyhidroxyalcanoate producing microorganisms. Electronic Journal of Biotechnology, 2007. 10(3): p. 348-357.

[26] Randriamahefa, S., et al., Fourier transform infrared spectroscopy for screening and quantifying production of PHAs by Pseudomonas grown on sodium octanoate. Biomacromolecules, 2003. 4(4): p. 1092-1097.

[27] Kansiz, M., et al., Fourier-transform infrared (FTIR) spectroscopy for monitoring and determining the degree of crystallisation of polyhydroxyalkanoates (PHAs). Analytical and bioanalytical chemistry, 2007. 388(5-6): p. 1207-1213.

[28] Hong, K., et al., A rapid method for detecting bacterial polyhydroxyalkanoates in intact cells by Fourier transform infrared spectroscopy. Applied Microbiology and Biotechnology, 1999. 51(4): p. 523-526.

[29] Galia, M.B., Isolation and analysis of storage compounds, in Handbook of hydrocarbon and lipid microbiology. 2010, Springer. p. 3725-3741.

[30] Linton, E., et al., Polyhydroxyalkanoate quantification in organic wastes and pure cultures using a single-step extraction and 1H NMR analysis. Water Science and Technology, 2012. 66(5): p. 10001006.

[31] Prabisha, T., et al., Production and characterization of PHB from a novel isolate Comamonas sp. from a dairy effluent sample and its application in cell culture. Biochemical Engineering Journal, 2015. 101: p. 150-159.

[32] Furrer, P., et al., Quantitative analysis of bacterial medium-chain-length poly ([R]-3hydroxyalkanoates) by gas chromatography. Journal of Chromatography A, 2007. 1143(1): p. 199206.

[33] Tan, G.-Y.A.Y., et al., Enhanced gas chromatography-mass spectrometry method for bacterial polyhydroxyalkanoates analysis. Journal of bioscience and bioengineering, 2014. 117(3): p. 379382

*Corresponding author.

E-mail address: ahmadidy2010@ gmail.com 